\title{
ENTERPRISE ENGINEERING - THE BASIS FOR SUCCESSFUL PLANNING OF E-BUSINESS
}

\author{
R. Jochem \\ Fraunhofer Institute for Production Systems and Design Technology \\ Pascalstrasse 8-9 \\ 10587 Berlin \\ GERMANY \\ roland.jochem@ipk.fhs.de
}

\begin{abstract}
Enterprise Modeling (EM) is currently in operation either as a technique to represent and understand the structure and behavior of the enterprise, or as a technique to analyze business processes, and in many cases as support technique for business process reengineering. However, EM architectures and methods for Enterprise Engineering can also be used to support the automation of business process for internet and e-business, because these new requirements need a clear, transparent and integrated definition and description of the business activities of the enterprise to be able to build up and operate an Enterprise Portal (Portal Engineering).
\end{abstract}

\section{INTRODUCTION}

In today's highly competitive global economy, the demand for high quality products manufactured at low costs with shorter cycle times has forced a number of manufacturing industries to consider various new product design, manufacturing, and management strategies. Recently, due to the rapid advances in Information Technology (IT), new paradigms have successively emerged such as e-business, automation of business processes to process orders with internet via Enterprise Portals. To cover these new requirements methods like Concurrent Engineering, Business Process Engineering and more Enterprise Engineering (EE) are needed.

Various architectures, methods and tools are available on the market, but there is a need for simple methods and tools for an integrated consideration of different reengineering and change management tasks, which save time and cost by reusing already represented information about the enterprise. There is also a need for modelbased components and reference models available as (commercial) building blocks to design, build and reengineer large systems. That components and reference models must be reusable and easily adaptable.

The requirements on flexible e-business-systems grow. Collaborative processes in the network of supplier, customer, provider and the company are more complex than processes within one company. Also the complexity of the related applications 
is high. The coordination of the different experts, department and the external suppliers and providers as well as the growing functionality of the systems cause additional complexity in the planning and introduction phase It doesn't work to consider only the technical aspects of e-business, it is more important to look at the required processes to use these systems in an efficient and successful way and to support the automation of these processes for making business via internet. Therefore projects for planning and introduction of e-business-systems within the enterprise require more and more a systematic process oriented procedure based on useful tools.

\section{ENTERPRISE ARCHITECTURES, METHODS AND TOOLS}

\subsection{Architectures and Methods}

Major research and development activities in the area of software engineering have resulted in following methods for system description and specification:

Information Modelling (Mertins, 1994): Entity Relationship (E/R); STEP/EXPRESS (especially for product data).

Functional and Process Modelling (Mertins, 1994): Structured Analysis (SA), Structured Analysis and Design Technique (SADT); Agent- Based Methods; PetriNet-based Methods; Process Specification Language (PSL).

Object Oriented Modelling (Jacobson, 1994; Coad, 1990): Object Oriented Software Engineering (Jacobsen); Object Oriented Analysis and Design (Coad/Yourdon); Unified Modelling Language (UML).

Major research and development programs for CIM have resulted in the following methods or architectures for enterprise modeling:

IDEF (ICAM Definition Method). It is made of a series of modeling methods comprising IDEF0 for functional modeling, IDEF1x/EXPRESS for information modeling, IDEF3 for business process modeling, IDEF4 for object modeling and IDEF5 for ontology modeling (Mayer, 1991).

GRAI-GIM. GIM (GRAI Integrated Methodology) is a methodology for design and analysis of production systems based on the GRAI method (Doumeingts, 1995). It includes modeling methods (GRAI grid, GRAI nets, IDEF0, MERISE) and focuses on decision system analysis of the enterprise.

CIMOSA (CIM Open System Architecture). It provides guidelines, architecture and an advanced modeling language for enterprise modeling covering function, information, resource and organization aspects of the enterprise (AMICE, 1999; Vernadat, 1996; Vernadat, 1998).

PERA (Purdue Enterprise Reference Architecture). This is a detailed methodology for enterprise engineering of industrial plants (Williams, 1997). It does not provide modeling constructs.

GERAM (Generalized Enterprise Reference Architecture and Methodology). It is a generalization of CIMOSA, GIM and PERA (IFAC-IFIP Task Force, 1997). It provides a methodology for enterprise engineering (PERA and GIM), a system life cycle (from PERA) and constructs for modeling (CIMOSA, 2001). 


\subsection{Tools for Modelling}

Many modeling products now exist on the market place (e.g. ARIS ToolSet, FirstSTEP, Bonapart, PrimeObject, or CimTool to name a few). Some of these packages are just modeling tools, others are more analysis tools and some even include a powerful simulation engine to evaluate performances of the system or analyze enterprise system behavior. Enterprise modeling is currently in operation either as a technique to represent and understand the structure and behavior of the enterprise, or as a technique to analyze business processes, and in many cases as support technique for business process reengineering (Vernadat, 1999).

Business processes and the relevant information should be described in one integral model. Information systems, the organisational structure, quality requirements and qualification requirements represent user views that are related to the core of the model. Process-organisational alternative and changes may then be evaluated with the regard to the effects on costs, quality, sytem support, organisational structure and the personnel-related qualification profile. The next chapters contain descriptions of a modelling language and a modelling tool that enable to create such an integrated model for the design of e-business processes (chapter 5) and for supporting the building of virtual enterprises.

\section{INTEGRATED ENTERPRISE MODELLING (IEM)}

The method employs the object-oriented approach to describe information and functions of objects as views on a single model of the system 'manufacturing company' integrally. The core of the model structure contains the views 'business process model' and 'information model'.

In the model the manufacturing processes and all activities that in reality are related to the production are described by functions and business processes that refer to certain objects.

The basis for the development of the model as a description of an individual company is formed by the object classes 'product', 'resource' and 'order'. The required corporate data and functions are assigned to these objects when creating the model. The relations between the objects are also determined. The result is that all tasks, the process organization, the corporate data, the production facilities and all components of the information system are registered comprehensively on any desired level of detail (Mertins, 1998-2).

The view 'business process model' emphasizes the tasks and business processes that are executed on the objects; the view 'information model' emphasizes the structures and features that describe objects. You are enabled to view one integrated model of the company from different angles. Business processes and the related information are described integrally in a model core. The information systems, the organizational structure, quality requirements and quality profiles constitute user views that relate to the model core. This enables you to evaluate processorganizational alternatives or modifications with regard to the effects on the control, the quality, the system support, the organizational structure and the staff's qualification profile (Mertins, 1996). 


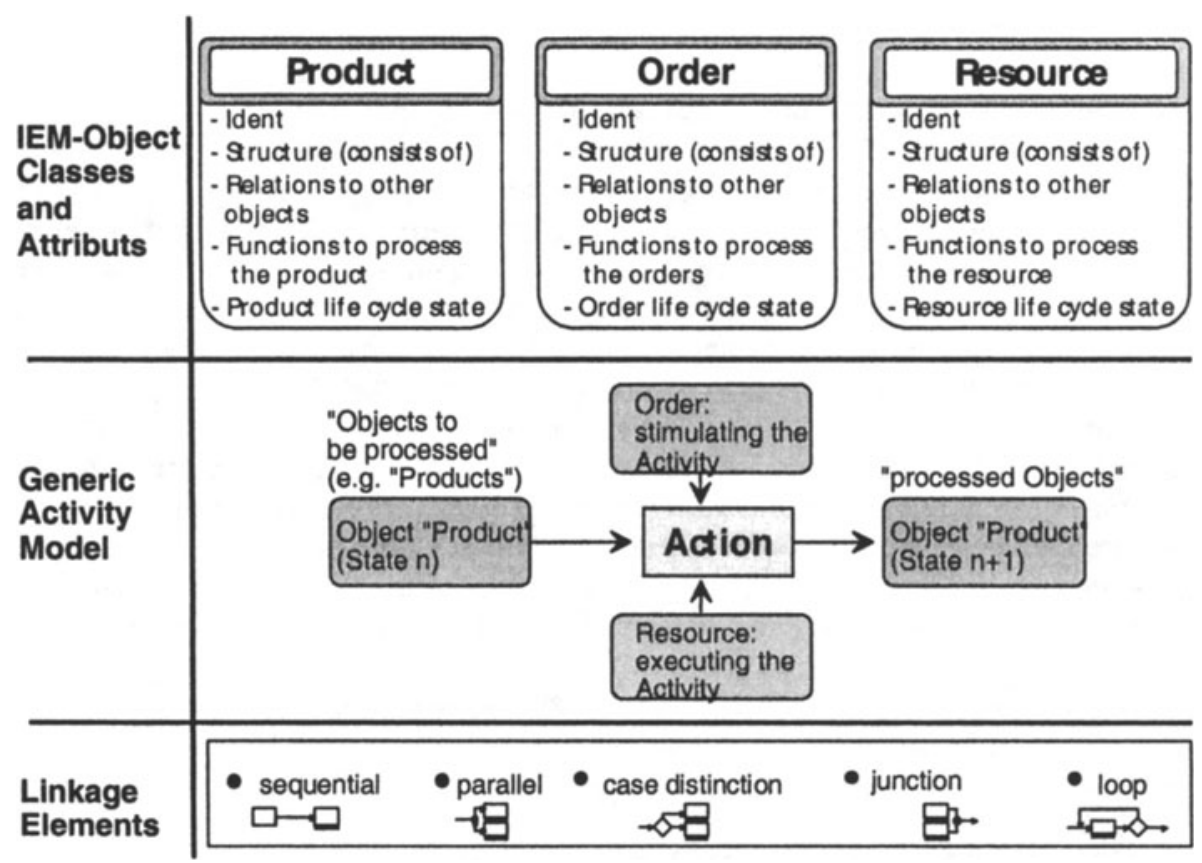

Figure 1 - IEM Modelling Elements

Figure 1 gives an overview of the modelling elements of IEM (Mertins, 1999). The object oriented modelling approach secures the reusability of modelling constructs and models for different purposes and enterprise types. Libraries of object classes and business processes can be created.

More than 50 industrial projects show, that the IEM methodology is easy to use and the resulting models bring transparency into the processes.

\section{SOFTWARE TOOL MOOGO}

The modeling tool MOOGO supports the method of object-oriented business process optimization. The universally usable tool to describe, analyze and optimize operational structures and business processes enables you to comfortably describe and purposively analyze products, resources, orders and the related business processes. Advantages of the use of the tool include the systematization of the planning and optimization processes and the reusability of the enterprise model for all projects and user views that concern corporate planning, such as information systems, controlling, quality management and organizational development. You may also generate standardized documents according to ISO $9000 \mathrm{ff}$. automatically. This reduces the certification process significantly. Class Libraries for specific application fields are available (e.g. ISO 9000 ff., ISO 14000ff., order processing, one-of-a-kind production etc.) (Mertins, 1999).

The tool enables you to reuse partial models and provides libraries with reference models. There are interfaces with the MS WINDOWS application programs WINWORD, EXCEL and ACCESS. Additionally predefined evaluation 
functions for standard queries on the model are provided by the tool. These functions can be parametrized by the user. Figure 2 shows the main components of the MOOGO system including the User Management (Mertins, 1998-1).

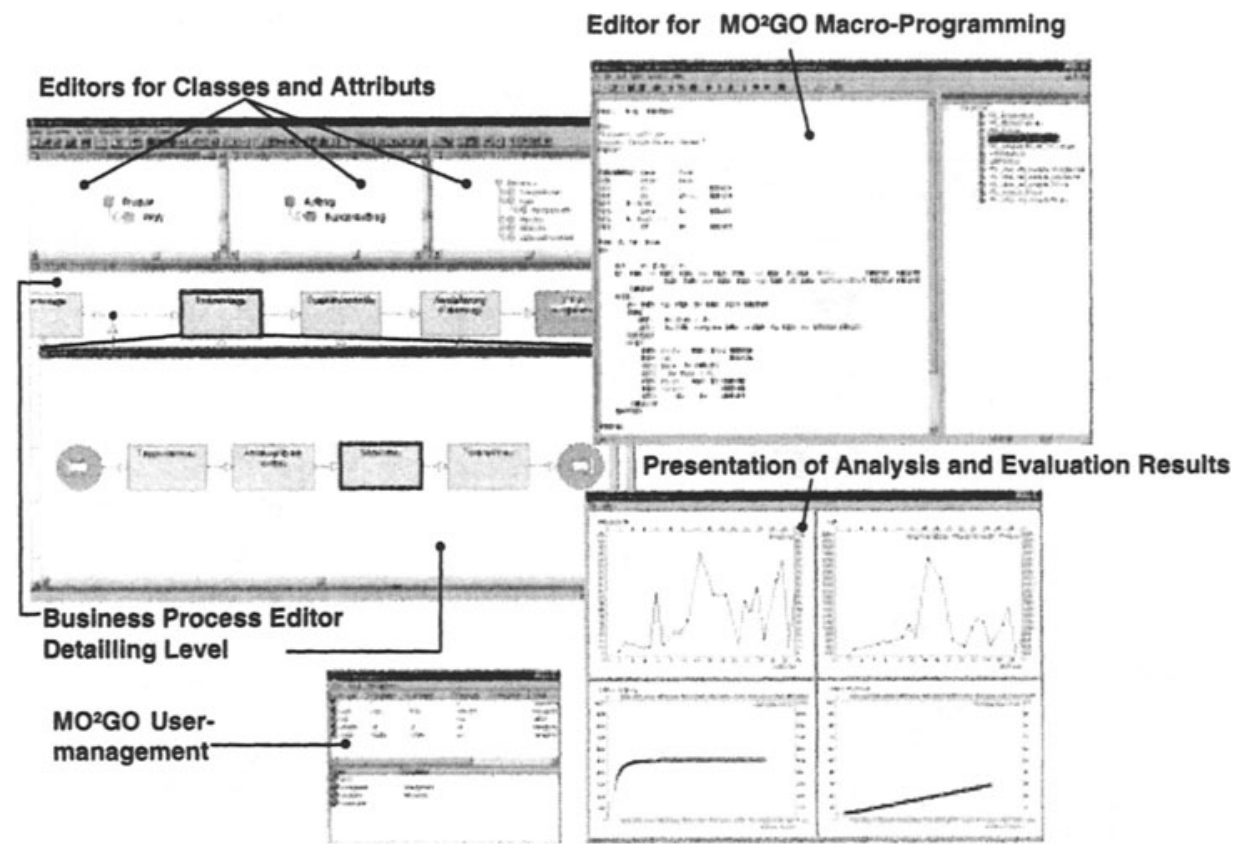

Figure 2 - Main Components of System MOOGO

\section{DESIGN OF PROCESSES FOR E-BUSINESS}

First of all the structure and architecture of the processes have to be changed for ebusiness. There are three new main processes described in a refernce model:

1 Enterprise development and control

2 Product or service offering via portal organized as Customer-SupplierRelationships

3 Support processes with an e-service backbone.

These main processes are modeled within the MOOGO-Tool by using the IEMMethodology. To speed up the modeling process an existing reference model "Order Processing" is used, where predefined typical modeling elements and model structures are described. Also "Best Practice" examples are available. It can be used to structure and optimize the order processing and order control processes with regard to processing times, cost structure, quality requirements, resource expenses and the support of information systems.

On a general level, the basic functions of order processing represent typical functions of order processing. The basic structures of order processing consist of these basic functions. 


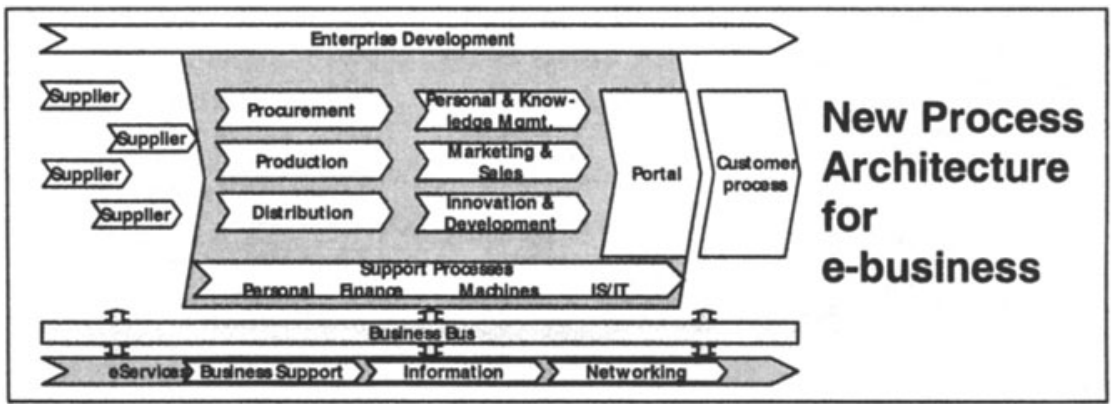

Representation of Process

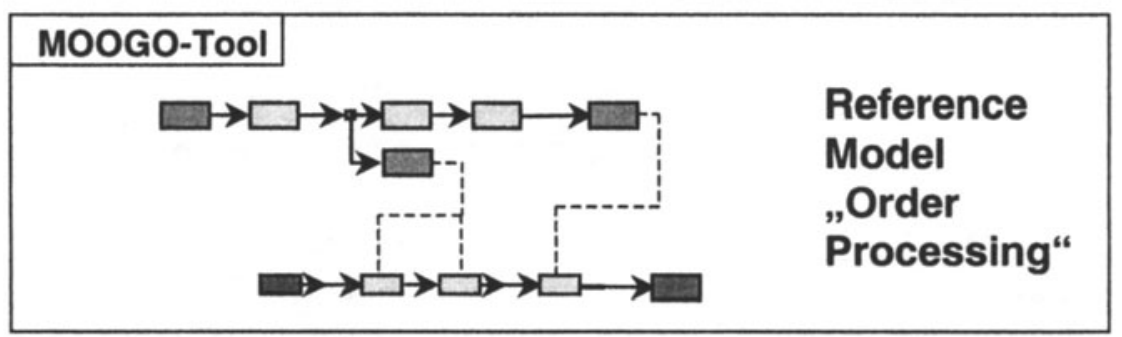

Adaption/Extension/Detailing of Model

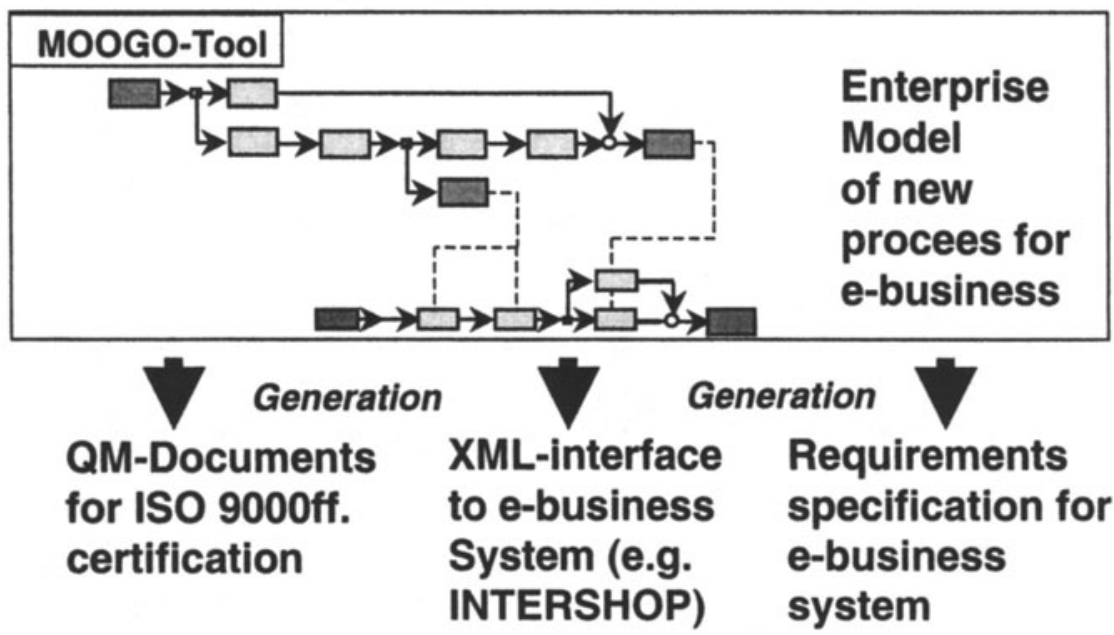

Figure 3 - Design of processes for e-business

The structures either represent typical processes, such as order preparation and tracking, or typical organizational forms of order control (centralized or decentralized, push or pull principle). The basic structures are used to model the order processing and control structure of the typical business process structure. These elements and structures are used and adapted during the model creation process for order processing via internet portal. 
When the model is build up in MOOGO the needed information is available to design the new processes for e-business by analysing different alternatives according to time, cost and quality parameters. MOOGO functionality allows (Figure 3)

1 the automated generation of Quality Management Documents for the ISO9000ff. Certification,

2 the connection to market available e-business-systems (e.g. INTERSHOP) via XML-interface,

3 the automated generation of a requirements specification for e-business-system selection, development or configuration.

\section{SUPPORTING THE PROCESS OF BUILDING VIRTUAL ENTERPRISES}

The core of virtual teamwork is communication, which is not only a technical issue but also a social and organisational one. Having the above described opportunities integrated in a tool like MOOGO, it is possible to support the entire process of bringing remote organisations together. This Virtual Enterprise (VE) formation process consists of the following steps:

1. Outsourcing (Publish tender online by supplied manufacturing standards, partner selection criteria or required competencies)

2. Define preliminary manufacturing process (use process modelling tool to identify all necessary processes and components)

3. Identify possible partners (by company competence profiles)

4. Select the best partner (based on process indicators)

5. Negotiation phase (based on detailed process and competence descriptions

During the entire formation process the tool MOOGO facilitates information exchange and negotiation between remote enterprises which want to establish a virtual partnership (B2B collaboration). The time span required for the formation of a virtual enterprise is reduced. It allows enterprises to from bidding syndicates or post open offers for the production of a particular component or product.

It is a chance for small and medium sized enterprises to increase development and production capacity by exploiting opportunities previously not identified due to limited information sharing and awareness of market opportunities. VE formation provides technological qualification and cost savings through specialisation, where enterprises hold a competitive advantage.

\section{CONCLUSION}

Systematic process oriented procedure based on useful tools is a prerequisite for the fast and efficient planning and introduction of e-business-systems. Not the technical aspects are the key success factors of e-business, but the organization of the collaborative business processes in the network of customer, supplier and the company in a efficient way, so that they are supported optimally by the innovative technology of the e-business-systems. This requires systematic e-business 
engineering. It is the basis for automation of business process for internet and the successful application of e-business-systems.

Today there is a need for simple methods and tools for the integrated consideration of different planning and reengineering tasks, which save time and cost by reusing already represented information about their enterprise.

The authors opinion is, that these methods and tools would be more used in any kind of enterprises if there was a standard description methodology like an unified enterprise modeling language (UEML) to be available on all modeling tools on the market. (IEM could be the basis for that; first attempt of ENV 12204 must be reworked and elaborated), and if there were model-based modules/components (e.g. based on reference models) available as (commercial) building blocks to design, build and reengineer enterprises (ENV, 1996; CIM-OSA, 2001) .

\section{REFERENCES}

AMICE. Enterprise Engineering and Integration - Why and How, a Primer on Concepts, Purpose and Business Value. CIM OSA Private Pubkication, 1999.

CIM-OSA. Enterprise Inter- and Intra-Organisational Integration - Need for International Consensus, $\mathrm{K}$. Kosanke, e-2001 European eBusiness \& eWork Conference, Venice, Italy, 2001.

Coad, P.; Yourdon, E. Object Oriented Analysis. Yourdon Press/Prentice Hall, Englewood Cliffs, NJ, 1990.

Doumeingts, G. Vallespir, B. A methodo-logy supporting design and implementation of CIM systems including economic evaluation. In Optimization Models and Concepts in Production Management (P. Brandimarte, A. Villa, eds.), Gordon \& Breach Science Publishers, NY, pp. 307-331, 1995.

ENV. ENV 12204. Constructs for Enterprise Modelling, 1996

ISO IS 15704. GERAM Generalized Enterprise Reference Architecture and Methodology, 1999.

Jacobson, I.; Ericsson, M.; Jacobson A. The Object Advantage. Addison-Wesley, 1994.

Mayer, R. Wholistic Design, Engineering, and Manufacture, Texas A\&M University, College Station, TX 77843, USA, 1991.

Mertins, K.; Süssenguth, W.; Jochem, R. Modellierungsmethoden fur rechnerintegrierte Produktionsprozesse (Hrsg.: G. Spur). Carl Hanser Verlag. München, Wien, 1994.

Mertins, K.; Jochem, R. Integrated Enterprise Modelling. In: Vernadat, F.: Enterprise Modelling and Integration, Chapman\&Hall, London, UK, 1996.

Mertins, K.; Jochem, R. MOOGO. In: Bernus, P., Mertins, K.; Schmidt, G.: Handbook on Architectures of Informations Systems, pp. 589-601, Springer Verlag, Berlin, Germany. 1998.

Mertins, K.; Jochem, R. Integrated Enterprise Modelling: Reference Architecture and Methodology. In: Handbook of Life Cycle Engineering: Concepts, Methods and Tools. Edited by Arturo Molina, José Manuel Sanchez, Andrew Kusiak. Chapman \& Hall, London, UK, 1998.

Mertins, K.; Jochem, R. Quality-Oriented Design of Business Processes. Kluwer Academic Publishers. Boston, London, Dordrecht. 1999.

Vernadat, F.B. Enterprise Modeling and Integration - Myth or Reality. In: Proceedings of CARS\&FOF 99 Conference, Aquas de Lindoia, Brazil, 1999

Vernadat, F.B. Enterprise Modeling and Integration: Principles and Applications, Chapman \& Hall, London, 1996.

Vernadat, F.B. The CIMOSA Languages. In (P. Bernus, K. Mertins and G. Schmidt, eds.) Handbook of Information Systems, Springer-Verlag, Berlin, pp. 243-263, 1998

Williams, T.J. The Purdue Enterprise Reference Architecture. Computers in Industry, 24 (2-3), 141, 1997. 